\title{
The whole moves less than the spin of its parts
}

\author{
Peter J. Kohler \\ Dartmouth College, Hanover, New Hampshire \\ Gideon P. CAPLovitz \\ Princeton University, Princeton, New Jersey \\ AND \\ Peter Ulric Tse \\ Dartmouth College, Hanover, New Hampshire
}

\begin{abstract}
When individually moving elements in the visual scene are perceptually grouped together into a coherently moving object, they can appear to slow down. In the present article, we show that the perceived speed of a particular global-motion percept is not dictated completely by the speed of the local moving elements. We investigated a stimulus that leads to bistable percepts, in which local and global motion may be perceived in an alternating fashion. Four rotating dot pairs, when arranged into a square-like configuration, may be perceived either locally, as independently rotating dot pairs, or globally, as two large squares translating along overlapping circular trajectories. Using a modified version of this stimulus, we found that the perceptually grouped squares appeared to move more slowly than the locally perceived rotating dot pairs, suggesting that perceived motion magnitude is computed following a global analysis of form. Supplemental demos related to this article can be downloaded from app.psychonomic-journals.org/content/supplemental.
\end{abstract}

It is often said that the whole is greater than the sum of its parts. Is this true for motion perception? Can a global-motion percept appear to be faster or slower than the sum of local-motion inputs from which it derives? For example, when four pairs of dots are rotated continuously around their respective pair centers, as is shown in Figure 1, the percept alternates between one of four independently rotating dot pairs (local motion) and one of two overlapping squares that have dots at their corners and that appear to translate along overlapping circular trajectories (global motion; Anstis, 2003). ${ }^{1}$ When the stimulus changes from the local to the global percept, it appears to move more slowly, even though the dots themselves never change speed at the level of the stimulus. This implies that the perceived speed of the global-motion percept is not determined solely by the local speeds of the individual dots, which is consistent with the view that motion is computed in light of the outputs of a stage of form analysis (Ames, 1951; Caplovitz \& Tse, 2007a, 2007b; Klopfer, 1991; Lorenceau \& Shiffrar, 1992; Shiffrar, Li, \& Lorenceau, 1995; Sinha \& Poggio, 1996; Tse, 2006; Tse \& Logothetis, 2002; Ullman, 1979). In the present article, we describe two psychophysical experiments that further examined the influence of perceived form on perceived motion.

\section{METHOD}

To investigate this "motion slowdown" effect, we effectively forced a local or global perceptual organization, rather than relying on the uncontrollable perceptual alternations that occur while one is viewing the original dot version of this stimulus (Anstis, 2003). To achieve this, we replaced each dot with an L-shaped stimulus (hereafter, "L"). ${ }^{2}$ In the global stimulus configuration, the Ls were oriented to align in such a manner that they would consistently induce the global percept (shown on the left side of Figure 2). In the local configuration, the orientations of the Ls were chosen pseudorandomly, so as to induce the local percept of four independently rotating pairs.

To further bias this configuration toward the local percept, the starting orientation of each pair was randomized on every trial (right side of Figure 2). We thus created two versions of the stimulus that, although almost identical at the local level (observing the motion of only a single $\mathrm{L}$ would not reveal whether one was observing the local or global configuration), were radically different in their ability to induce the global percept.

Stimuli were white $\left(67.02 \mathrm{~cd} / \mathrm{m}^{2}\right)$ on a gray $\left(4.09 \mathrm{~cd} / \mathrm{m}^{2}\right)$ background, with a white $\left(67.02 \mathrm{~cd} / \mathrm{m}^{2}\right)$ vertical bar separating the two sides of the display. The two line segments that constituted each $\mathrm{L}$ measured $0.5^{\circ}$ of visual angle in length. Within a pair, the distance between Ls measured $0.8^{\circ}$ (see Figure 2). The vertical distance between pairs was $3^{\circ}$, and the horizontal distance between pairs was $4^{\circ}$. Each stimulus configuration was centered $6.84^{\circ}$ from the fixation spot. While maintaining central fixation, observers reported that they could easily see all the moving elements, and they consistently

G. P. Caplovitz, gcaplovi@princeton.edu 


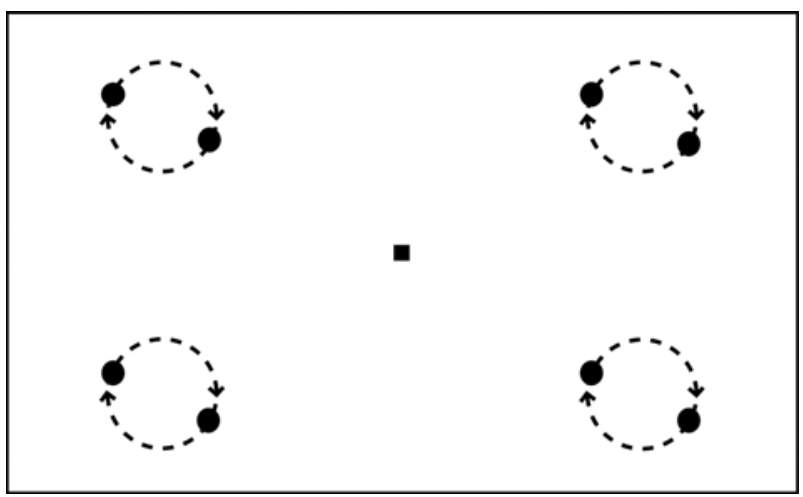

Figure 1. Stuart Anstis's original stimulus.

reported global-motion percepts in the aligned configuration, and local-motion percepts in the unaligned configurations.

In the main experiment (Experiment 1), one global stimulus configuration (standard) was always presented with the pairs of Ls rotating at the same angular velocity $\left(57.6^{\circ}\right.$ of rotation per sec) on every trial. This reference speed was chosen because it permitted the perception of both local and global percepts. The standard was presented randomly to either the left or the right side of the fixation spot. Another stimulus (test) was presented simultaneously to the opposite side, with L pairs rotating at 1 of 11 different possible angular velocities selected around the reference angular velocity $\left(17.3^{\circ} \%\right.$ sec, $28.8^{\circ} / \mathrm{sec}, 40.3^{\circ} / \mathrm{sec}, 46.1^{\circ} / \mathrm{sec}, 51.9^{\circ} / \mathrm{sec}, 57.6^{\circ} / \mathrm{sec}, 63.4^{\circ} / \mathrm{sec}$, $69.2^{\circ} / \mathrm{sec}, 74.9^{\circ} / \mathrm{sec}, 86.4^{\circ} / \mathrm{sec}$, or $98.0^{\circ} / \mathrm{sec}$ ). On each trial, the test could be either the global or the local configuration. Stimuli were presented for $1.5 \mathrm{sec}$ and then removed from the screen. Observers were instructed to indicate, via a $2 \mathrm{AFC}$ buttonpress, whether the stimulus on the left or the stimulus on the right was moving faster. Each possible test speed was presented in a pseudorandom order, 20 times for both the local and global test conditions. The direction of rotation of each pair of Ls was the same for each pair on a given trial, but it was randomized across trials.

Observers were required to maintain central fixation while the stimuli were on the screen. Fixation was monitored using an SR Research eyelink 2 head-mounted eyetracker. If the point of fixation moved more than $1.5^{\circ}$ away from the central fixation spot while the stimuli were displayed, the trial was aborted and was started again once central fixation had been regained.

A second experiment using the same participants (Experiment 2) was run to control for the potential that local differences in motion signals were produced because Ls were oriented differently across the two configurations. This experiment was the same as the one that was described above, except that only a single pair of Ls was presented in each configuration, equidistant from fixation at mirroropposite positions around the vertical midline. The two configurations consisted of a pair of adjacent Ls from either the local or the global configurations of Experiment 1. Experiment 2 served as a necessary control, because no global percept of squares is possible when there is only one L pair. Any differences that were found between the two conditions in Experiment 2 must have been due to differences that arose from the orientation of the Ls alone. Conversely, if these two control conditions were perceived to be moving at the same speed, then any differences that were observed in Experiment 1 must have been due to global versus local perceptual organization and not to local differences in the orientations of the Ls.

Nine paid observers, who were naive about the purpose of the study (each gave informed written consent, according to the guidelines of the internal review board of Dartmouth College, prior to participating), participated in the experiments. For each of the 9 observers, we calculated the proportion of trials in which the test stimulus appeared to move faster than the standard, for both the global and local test stimuli. The data from each observer were then fit using a logit function in MATLAB, from which a point of subjective equality (PSE) was computed, corresponding to the speed at which the test needed to move in order to be perceived as having the same speed as the standard.

\section{RESULTS}

The perceptually grouped global configuration was perceived to move more slowly than the local configuration in Experiment 1 (Figure 3). For a local test stimulus to be perceived as moving at the same speed as the global standard, the rotating pairs had to rotate significantly more slowly [mean observer PSE $=52.07 ; t(8)=$ $-2.355, p=.046]$ than $57.6^{\circ} / \mathrm{sec}$, which is the speed at which the pairs in the global standard rotated, corresponding to a speed shift of $9.6 \%$. This was not the case for the global test stimulus [mean PSE $=59.70 ; t(8)=$ $1.226, p=.255]$.

In Experiment 2, however, no difference in perceived speed was observed for the standard and two test configurations [global-test mean PSE $=58.44 ; t(8)=1.280, p=$ .236; local-test mean PSE $=56.25 ; t(8)=-1.653, p=$ .137]. Although we refer here to these configurations as "local" and "global," it is important to note that in neither case was a global percept perceived. A repeated measures ANOVA $^{3}$ on the PSEs from the two experiments (see Figure 3), with the number of Ls as one factor and local or global configuration as the other factor, revealed a significant interaction $\left[F(1,8)=8.111, p=.022 ; \eta_{\mathrm{p}}^{2}=.503\right]$ between number of Ls and stimulus type. This indicates that the global-motion percept appeared to move more slowly than the local-motion percept, and that this cannot be explained solely on the basis of local differences in the motion signals arising from the different $\mathrm{L}$ orientations.

\section{DISCUSSION}

Our results provide empirical evidence that the global configuration appears to move more slowly than the local configuration. Because actual local motions of individual Ls that were considered in isolation were identical across local and global percepts, speed perception cannot

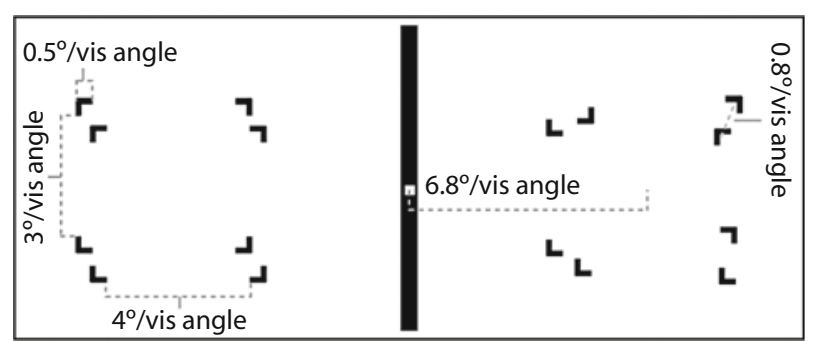

Figure 2. The stimulus. On the left side, the Ls are arranged as "corners" of virtual squares, so that a global percept of two overlapping, translating squares is facilitated. On the right side, the Ls are pseudorandomly arranged, so that a local percept is facilitated, with pseudorandom pair organizations/orientations. Stimulus colors were different in the actual experiment. 
A

Experiment 1

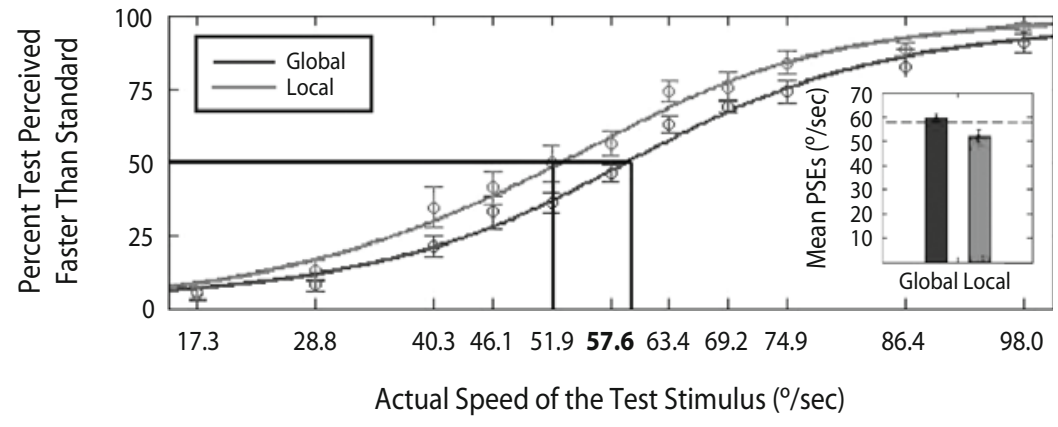

B

Experiment 2

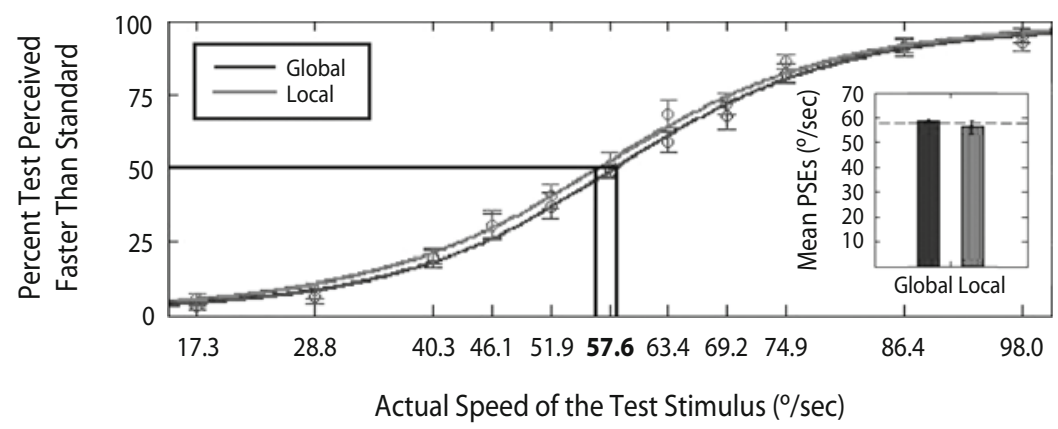

Figure 3. (A) Results of Experiment 1. Percentage of responses in which the observers reported that the speed of the test stimulus was faster than that of the standard stimulus, plotted as a function of the actual physical speed of the test stimulus. The open circles represent the mean performance across participants; the curves are fit to these means. (blue, global test; red, local test). The black lines indicate the test speed that was necessary for both stimuli to be perceived as moving at the same speed (PSE). Insets show mean PSEs, individually computed for each participant for both test conditions; the dashed line indicates the actual speed of the standard (always $57.6 \%$ bold, on the main $x$-axis). (B) Results of Experiment 2. Note that the difference in PSE between the local and global test conditions in Experiment 1 was significantly greater than that in Experiment 2. (Error bars represent the standard errors of the means.)

be determined solely on the basis of local speed-tuned mechanisms. Rather, motion appears to be computed in light of input from a global analysis that interpolates forms such as the virtual squares that were perceived in the global configuration (Ames, 1951; Caplovitz \& Tse, 2006, 2007a, 2007b; Klopfer, 1991; Lorenceau \& Shiffrar, 1992; Shiffrar et al., 1995; Sinha \& Poggio, 1996; Tse, 2006; Tse \& Logothetis, 2002; Ullman, 1979). Instantaneous motion vectors must be computed in light of the outputs of such nonlocal grouping or segmentation operations. These form-construction operations in turn are known to take input from the motion system, as in "common fate" grouping (see, e.g., Uttal, Spillmann, Stürzel, \& Sekuler, 2000) or the computation of formfrom-motion (see, e.g., Britten, Newsome, \& Saunders, 1992; Schoenfeld et al., 2003).

Why the global-motion percept was perceived to move more slowly than the local percept remains unclear, but the literature has suggested that perceived motion magnitude may be influenced by the following factors: (1) size, (2) number, and (3) emergent motion signals. We consider each in turn.
(1) Size: Using dark figures that were presented on a white background and were viewed through $15 \times 5 \mathrm{~cm}$ apertures, Brown (1931) found that the perceived velocity of a moving object decreased as its size increased. It is possible that the illusory squares of the global percept were treated as unified objects by the motion-processing system. Since the squares were larger than the L pairs, this could have caused a decrease in perceived velocity of the squares, relative to that of the $\mathrm{L}$ pairs.

(2) Number: It has been suggested that speed discrimination is in part dependent on the parsing of an image into discrete entities and the pooling of information across them over space and time. Spatially separated drifting gratings, when they are perceptually grouped and perceived as one large, partially occluded grating, have increased speed discrimination thresholds. This is thought to occur because an increase in the number of samples from which to estimate speed increases sensitivity to speed (Verghese \& Stone, 1996). The global percept here may be seen to move more slowly because of an increase in speed-discrimination threshold, which is caused by the parsing of the Ls into two large squares in the global 
condition, rather than into eight translating individual Ls or four rotating L pairs, as in the local condition. This is consistent with the idea that perceptual grouping or parsing processes that must operate after, or at least in parallel with, the detection of local motion signals influence the speed of the perceived motion.

Similarly, if two directions or speeds of motion are presented simultaneously using dynamic random dots, the percept is one of two transparent surfaces (Watamaniuk, Flinn, \& Stohr, 2003), suggesting that motion perception may rely upon at least two stages of processing. In the first stage, differential sources of motion information are segmented from each other. Many factors may contribute to this segmentation process, including the principles of perceptual grouping and the correlations between different local motion signals over global space (Weiss \& Adelson, 2000 ). In the second stage, the local-motion signals within each segmented group are integrated to form the perceived global direction, translational speed, and angular velocity. The resultant motion percept likely depends on the spatiotemporal relationships between the individual elements within each segmented group. In this framework, unlike in the spatial pooling hypothesis (Verghese \& Stone, 1996), the perceived motion of each segmented group is largely uninfluenced by the motions of other groups in the image (Weiss \& Adelson, 2000).

(3) Emergent motion signals: A number of different speed illusions show that different types of complex global motion yield different speed percepts, although the local motions remain identical. Geesaman and Qian $(1996,1998)$ found that dots that were moving in an expanding pattern were seen to move faster than identi$\mathrm{cal}$ dots that were moving in a rotating pattern. Bex and Makous (1997) likewise found that a radial grating was perceived to move faster than a vertical grating, although the local speed components were identical. Additionally, Bex, Metha, and Makous (1998) found that the speeds of translational and rotational motion patterns were perceived to be identical, whereas radial patterns appeared to move approximately one third faster. One explanation for these effects is that neurons in MSTd have a bias for expansion, thus yielding the difference in perceived speed (Geesaman \& Qian, 1996); however, it has been shown that contraction, as well as expansion, is experienced as moving faster than rotation, which would not be predicted by the model, since the numbers of contractionand rotation-sensitive cells in MSTd are equal (Clifford, Beardsley, \& Vaina, 1999). Furthermore, no difference has been found in speed discrimination among expansion, contraction, and rotation, which would be expected if one type of movement leads to lesser activation than do the other two (Clifford et al., 1999). Instead, it has been suggested that the effect arises because of mechanisms that are specific to optic flow (Bex \& Makous, 1997), because expansion and contraction can be interpreted as motion in depth and thus are subject to constraints other than rotation (Clifford et al., 1997). In the present experiments, an emergent motion signal that was associated with object rotation might have contributed to the effect, because emergent rotation is present in the local, but not the global, case.

A related possibility is that the rotational motion of the Ls in the local case did not optimally activate specialized rotation-sensitive motion detectors. For rotating dots, if dot spacing is small (approximately less than $0.5^{\circ}$ ), angular velocity will determine the speed percept, whereas for a larger spacing, speed perception will be based on linear velocity (Barraza \& Grzywacz, 2002; Caplovitz \& Tse, 2007a). Because the spacing between the corners of the Ls was larger than in the present experiments, perceived motion in the local condition might have been based on linear translation, yielding an imprecise rotational magnitude signal.

In addition, it has been found that a thin ellipse is perceived to rotate faster than a fat ellipse, even when they are in fact rotating at the same angular velocity (Caplovitz, Hsieh, \& Tse, 2006). The same effect of aspect ratio on perceived angular velocity was observed for ellipses that were defined not by continuous contours, but by a number of equally spaced dots (Caplovitz \& Tse, 2007a). Importantly, when the dots were randomly distributed so that no elliptical contours could be formed, but the dots still had local motions that were identical to those that were used to form the ellipses, no effect of form on perceived angular velocity was observed. Thus, the perceived angular velocity of the rotating dotted ellipses was driven by the emergent motion signals that arose from the virtual contours of the ellipses that were formed by perceptual grouping processes. Similar mechanisms could lead rotating pairs of discrete elements to appear to move faster than translating squares. Moreover, it has been found that drifting lines that are not orthogonal to their direction of motion appear to travel more slowly than those that are (Castet, Lorenceau, Shiffrar, \& Bonnet, 1993; ScottBrown \& Heeley, 2001). The emergent motion signal that was observed in the global condition of the present study, that of two squares translating along a circular trajectory, can be equated to that of 8 drifting lines, which are rarely orthogonal to their direction of motion. Similarly, in the local case, the rotating Ls could have been seen as 16 short drifting lines -8 vertical and 8 horizontal. This could explain the effect, since (1) in the global case, the (illusory) lines were longer and thus would have had a stronger effect (Castet et al., 1993), and (2) groups of short lines do not show an enhanced effect unless they are arranged in a colinear manner (Scott-Brown \& Heeley, 2001), which was not the case here.

In conclusion, the global percept of translating squares was perceived to move more slowly than the local percept of rotating pairs of Ls, even though the local motion signals in the image were identical in both perceptual organizations. This result therefore draws a clear distinction between the stages of processing that are involved in the detection of visual information that is explicitly represented in the image, and the stages of perceptual construction that can extract and even create information that is not explicit in the image. Perceived motion magnitude is dictated by emergent form and motion signals (not available 
in the image) that are constructed on the basis of detectedmotion signals (which are available in the image). Future models of motion processing, therefore, cannot rely solely on local analysis of motion energy signals, and must take into account a stage of global form analysis and the emergent motion signals of constructed contours, corners, and other emergent form features.

\section{AUTHOR NOTE}

This work was supported by National Science Foundation Grant NSF BCS- 0545303 to P.U.T. and National Science Foundation Predoctoral Fellowship 2005031192 to G.P.C. Correspondence concerning this article should be addressed to G. P. Caplovitz, Department of Psychology, 3-N-13 Green Hall, Princeton University, Princeton, NJ 08544 (e-mail: gcaplovi@princeton.edu).

\section{REFERENCES}

AMES, A. (1951). Visual perception and the rotating trapezoidal window. Psychological Monographs, 65, 1-32.

ANstis, S. (2003). Levels of motion perception. In L. Harris \& M. Jenkin (Eds.), Levels of perception (pp. 75-99). New York: Springer.

BARRAZA, J. F., \& GRZYWACZ, N. M. (2002). Measurement of angular velocity in the perception of rotation. Vision Research, 42, 2457-2462. doi:10.1016/S0042-6989(02)00259-6

Bex, P. J., \& Makous, W. (1997). Radial motion looks faster. Vision Research, 37, 3399-3405. doi:10.1016/S0042-6989(97)00088-6

Bex, P. J., Metha, A. B., \& Makous, W. (1998). Psychophysical evidence for a functional hierarchy of motion processing mechanisms. Journal of the Optical Society of America A, 15, 769-776. doi:10.1364/ JOSAA.15.000769

Britten, K. H., Newsome, W. T., \& Saunders, R. C. (1992). Effects of inferotemporal cortex lesions on form-from-motion discrimination in monkeys. Experimental Brain Research, 88, 292-302.

BRown, J. F. (1931). The visual perception of velocity. Psychologische Forschung, 14, 199-232.

Caplovitz, G. P., Hsieh, P.-J., \& Tse, P. U. (2006). Mechanisms underlying the perceived angular velocity of a rigidly rotating object. Vision Research, 46, 2877-2893. doi:10.1016/j.visres.2006.02.026

CaPlovitz, G. P., \& Tse, P. U. (2006). The bar-cross-ellipse illusion: Alternating percepts of rigid and nonrigid motion based on contour ownership and trackable feature assignment. Perception, 35, 993-997.

Caplovitz, G. P., \& Tse, P. U. (2007a). Rotating dotted ellipses: Motion perception is driven by grouped figural rather than local dot motion signals. Vision Research, 47, 1979-1991. doi:10.1016/j .visres.2006.12.022

CaPlovitz, G. P., \& Tse, P. U. (2007b). V3A processes contour curvature as a trackable feature for the perception of rotational motion. Cerebral Cortex, 17, 1179-1189. doi:10.1093/cercor/bhl029

Castet, E., Lorenceau, J., Shiffrar, M., \& Bonnet, C. (1993). Perceived speed of moving lines depends on orientation, length, speed and luminance. Vision Research, 33, 1921-1936. doi:10.1016/0042 -6989(93)90019-S

Clifford, C. W. G., Beardsley, S. A., \& Vaina, L. M. (1999). The perception and discrimination of speed in complex motion. Vision Research, 39, 2213-2227. doi:10.1016/S0042-6989(98)00314-9
Geesaman, B. J., \& Qian, N. (1996). A novel speed illusion involving expansion and rotation patterns. Vision Research, 36, 3281-3292. doi:10.1016/0042-6989(96)00054-5

Geesaman, B. J., \& Qian, N. (1998). The effect of complex motion pattern on speed perception. Vision Research, 38, 1223-1231. doi:10.1016/S0042-6989(97)00279-4

KLOPFER, D. S. (1991). Apparent reversals of a rotating mask: A new demonstration of cognition in perception. Perception \& Psychophysics, 49, 522-530.

LORENCEAU, J., \& Shiffrar, M. (1992). The role of terminators in motion integration across contours. Vision Research, 32, 263-273.

Schoenfeld, M.A., WoldorfF, M., Düzel, E., Scheich, H., Heinze, H.-J., \& MANGUN, G. R. (2003). Form-from-motion: MEG evidence for time course and processing sequence. Journal of Cognitive Neurosciences, 15, 157-172. doi:10.1162/089892903321208105

Scott-Brown, K. C., \& Heeley, D. W. (2001). The effect of the spatial arrangement of target lines on perceived speed. Vision Research, $\mathbf{4 1}$, 1669-1682. doi:10.1016/S0042-6989(01)00042-6

Shiffrar, M., Li, X., \& Lorenceau, J. (1995). Motion integration across differing image features. Vision Research, 35, 2137-2146. doi:10.1016/0042-6989(94)00299-1

Sinha, P., \& Poggio, T. (1996). Role of learning in three-dimensional form perception. Nature, 384, 460-463.

TsE, P. U. (2006). Neural correlates of transformational apparent motion. NeuroImage, 31, 766-773. doi:10.1016/j.neuroimage.2005.12.029

Tse, P. U., \& Logothetis, N. K. (2002). The duration of 3-D form analysis in transformational apparent motion. Perception \& Psychophysics, 64, 244-265.

Ullman, S. (1979). The interpretation of visual motion. Cambridge, MA: MIT Press.

Uttal, W. R., Silllmann, L., Stürzel, F., \& Sekuler, A. B. (2000). Motion and shape in common fate. Vision Research, 40, 301-310. doi:10 .1016/S0042-6989(99)00177-7

Verghese, P., \& Stone, L. S. (1996). Perceived visual speed constrained by image segmentation. Nature, 381, 161-163. doi: $10.1038 / 381161 \mathrm{a} 0$

Watamaniuk, S. N. J., Flinn, J., \& Stohr, R. E. (2003). Segregation from direction differences in dynamic random-dot stimuli. Vision Research, 43, 171-180. doi:10.1016/S0042-6989(02)00434-0

Weiss, Y., \& Adelson, E. H. (2000). Adventures with gelatinous ellipses-Constraints on models of human motion analysis. Perception, 29, 543-566.

\section{NOTES}

1. See Supplementary Demo 1.

2. See Supplementary Demo 2.

3. Mauchly's test indicated that the assumption of sphericity had not been violated.

\section{SUPPLEMENTAL MATERIALS}

Demos of the motion described in this article, created in .mov format for Quicktime, can be downloaded from app.psychonomic-journals.org/ content/supplemental.

(Manuscript received October 28, 2008; revision accepted for publication December 21, 2008.) 